\title{
From a face to its category via a few information processing states in the brain
}

\author{
Marie L. Smith, ${ }^{\mathrm{a}, \mathrm{b}}$ Frédéric Gosselin, ${ }^{\mathrm{c}}$ and Philippe G. Schyns ${ }^{\mathrm{a}, \mathrm{b}, *}$ \\ ${ }^{a}$ Centre for Cognitive Neuroimaging (CCNi), University of Glasgow, 58 Hillhead Street, Glasgow G12 8QB, UK \\ ${ }^{\mathrm{b}}$ Department of Psychology, University of Glasgow, 58 Hillhead Street, Glasgow G12 8QB, UK \\ ${ }^{\mathrm{c}}$ Department de Psychologie, Université de Montréal, CP 6128, succ. Centre-ville, Montréal, Canada H3C $3 J 7$
}

Received 12 March 2007; revised 18 April 2007; accepted 9 May 2007

Available online 25 May 2007

\begin{abstract}
Cognitive neuroscience assumes a correspondence between specific spatio-temporal patterns of neural activity and the states of a mechanism that processes cognitive information. Mechanistic explanations of cognition should therefore translate patterns of neural activity into the components of a formal mechanism: a set of information processing states and their transitions. For the first time, we carried out this research programme with four naive observers instructed to categorise randomly presented face information. With classification image techniques, we revealed the diagnostic features that the brain requires to produce correct behaviour (i.e., two eyes for gender categorisation in one session; the mouth for expression in the other session). With the same techniques applied to brain signals, we revealed the features processing states associated with modulations of oscillatory EEG energy (measured on occipito-temporal face-sensitive electrodes). Here we show how transitions between distinct feature processing states in the theta/alpha $[4-12 \mathrm{~Hz}]$ oscillatory bands implement two face categorisations. On the left and right occipito-temporal electrodes of each observer, processing of the contra-lateral eye precedes bilateral integration of the features required for behaviour. For the first time, we relate stimulus information to behaviour via sequences of categorisation-specific feature processing states in the brain.
\end{abstract}

(C) 2007 Elsevier Inc. All rights reserved.

To study face categorisation mechanisms in an information system such as the brain, automata theory (Hopcroft and Ullman, 1979) specifies three generic questions that must be addressed to relate brain activity to the states of an information processing mechanism (i.e., a formal automaton). The first question is that of form: what is the nature of the brain activity supporting face processing - i.e., the states of the brain correlated with face processing? The second question is that of content: what is the information content processed in these brain states? The third question is that of transition:

\footnotetext{
* Corresponding author. Department of Psychology, University of Glasgow, 58 Hillhead Street, Glasgow G12 8QB, UK.

E-mail address: philippe@psy.gla.ac.uk (P.G. Schyns).

Available online on ScienceDirect (www.sciencedirect.com).
}

how does information flow from one brain state to the next between stimulus onset and behavioural response? So far, cognitive neuroimaging studies have not simultaneously addressed these three questions. Until this is resolved, inferences about cognitive information processing mechanisms will be severely restricted.

To illustrate the problem, researchers have proposed that slow brain rhythms could form distinct states of information processing, on the basis of considerable evidence of a consistent relationship between low frequency, theta $(4-8 \mathrm{~Hz})$ and alpha $(8-12 \mathrm{~Hz})$, oscillatory EEG activity of the neural substrate and behavioural variables (Van Rullen and Koch, 2003; Ward, 2003, for reviews). However, definitive evidence is lacking in part because it has been proven difficult to identify precisely the information content of these states from the parametric modulations (i.e., phase and amplitude) of slow brain rhythms. Consequently, little is known about the detailed flow of information (i.e., information processing states and their transitions) that the brain goes through when it dynamically evolves through peaks and troughs at a given rhythm to implement the information processing mechanisms of cognition.

Here, we adopted an established system modeling approach: build a mathematical model with the components of a finite state automaton. A finite state automaton comprises a finite number of information processing states together with conditions on state transitions specified in a table. We extracted a finite set of information processing states from the oscillatory EEG and then revealed their transitions (the flow of information), which reflect the underlying computations of information from the input face in order to produce categorisation behaviour. ${ }^{1}$

Specifically, four observers resolved two biologically relevant face categorisations (male-female, GENDER; expressive or not, EXNEX) of the same stimulus set (i.e., males and females with two

\footnotetext{
${ }^{1}$ We are aware that "mechanism" is a loaded term, which is why we limit its scope to the formal definition given in the theory of computation (Hopcroft and Ullman, 1979): a finite set of information processing states together with a table specifying the state transitions that implement a particular computation. Note that this definition does not associate a mechanism with a particular level of neural integration.
} 
expressions: smiling and neutral). For each observer and categorisation (GENDER and EXNEX), the experiment established a oneto-one correspondence between random samples of facial information presented on each single trial and response to this information-i.e., behavioural responses and the EEG measured on face-sensitive electrodes, the left and right low occipito-temporal electrodes (Smith et al., 2004). With classification image techniques (Gosselin and Schyns, 2001), we estimated across the 4000 trials of each task how facial information modulated responses. For the EEG, we examined modulations of oscillatory energy in a Time $\times$ Frequency domain: every $4 \mathrm{~ms}$ from -200 to $600 \mathrm{~ms}$ around stimulus onset; every $2 \mathrm{~Hz}$ from 4 to $30 \mathrm{~Hz}$. For behaviour we examined categorisation accuracy and reaction time, see Fig. 1.

\section{Methods}

Subjects

Subjects were four paid observers (BB, MB, ML and RZ) from Glasgow University, UK.

\section{Stimuli}

Face stimuli were computed from $256 \times 256$ pixels grey-scale pictures of 10 actors ( 5 males and 5 females) each displaying two facial expressions (neutral and happy). All photographs were taken under standardised conditions of illumination and hairstyle was normalised across faces to eliminate this feature. Stimuli were presented on a light grey background at the centre of a computer monitor with a fixed chin rest maintaining a constant $1-\mathrm{m}$ viewing distance, resulting in each stimulus spanning $4.6 \times 4.6^{\circ}$ of visual angle.

\section{Procedure}

A trial started with the 500-ms presentation of a fixation cross spanning $0.4^{\circ}$ of visual angle. It was immediately followed by a randomly selected face picture whose information was revealed through 14 two-dimensional Gaussian apertures $\left(\sigma=0.22^{\circ}\right.$ of visual angle) randomly allocated with the constraint that each aperture remained within the area of the face (see Fig. 1, Stimuli). Previous experiments determined that 14 apertures are required to reach a minimum of $75 \%$ correct categorisations in the GENDER and EXNEX tasks (Gosselin and Schyns, 2001). The sparse face remained on screen for $1500 \mathrm{~ms}$ and observers were instructed to respond as quickly as possible without making mistakes by depressing the appropriate button of a two-key response box. In one 4000 trials session observers indicated the gender of each sparse face and in a second session they indicated whether the sparse face was expressive or not. Order of task was counterbalanced across observers. Short breaks were allowed every 100 trials.

\section{EEG recording}

Scalp electrical activity (EEG) was recorded with 32 (ML and $\mathrm{BB}) / 64$ (MB and RZ) sintered silver/silver chloride electrodes mounted in an electrode cap (Quick-Cap/Easy-Cap respectively) at electrode positions including the standard 10-20 system positions along with intermediate positions (and an additional row of low occipital electrodes in the Easy-Cap). The right mastoid (TP10)/ linked mastoids served as initial common reference, and the $\mathrm{AFz}$ electrode as ground. Vertical electro-oculogram (vEOG) was bipo- larly registered above and below the dominant eye and horizontal electro-oculogram (hEOG) at the outer canthi of the eyes. Signals were continuously acquired at 250/1024 Hz. Electrode impedance was kept below $10 \mathrm{k} \Omega$ throughout. Analysis epochs were generated offline starting $200 \mathrm{~ms}$ prior to stimulus onset and continuing for $600 \mathrm{~ms}$, aligned to a 200-ms pre-stimulus baseline. EEG and EOG artifacts were removed by using a $[-30 \mu \mathrm{V} ;+30 \mu \mathrm{V}]$ deviation threshold over 200-ms intervals on all electrodes. (Note that the EOG rejection procedure will reject rotations of the eyeball from $0.9^{\circ}$ inward to $1.5^{\circ}$ downward of visual angle, when the stimulus spanned $4.6 \times 4.6^{\circ}$ of visual angle). Artifact free trials were low pass filtered at $30 \mathrm{~Hz}$ and re-referenced to average reference (excluding the EOG channels, see Schyns et al., 2003 and Smith et al., 2004, for details). For each subject we choose the left and right hand side lateralised occipito-temporal electrodes exhibiting the largest amplitude variation in the $300 \mathrm{~ms}$ following stimulus onset. We analysed occipito-temporal electrodes P9 and P10 for ML, P7 and P10 for BB, PO5 and PO8 for $\mathrm{MB}$ and P7 and P8 for RZ. Henceforth we refer to PO5, P7, P9 as occipito-temporal left (OTL) and $\mathrm{P} 8, \mathrm{PO} 8$ and $\mathrm{P} 10$ as occipito-temporal right (OTR).

\section{Computation: Behavioural Classification Image}

Accuracy: On each trial of a categorisation task, the 14 randomly located Gaussian apertures make up a two-dimensional mask, a 'bubble mask', that reveals a sparse face (see Fig. 1, Stimuli). Observers will tend to be correct if this information is diagnostic for the categorisation at hand. Across trials, we computed a probability of being correct-i.e., we summed the aperture masks leading to correct categorisations and divided the result by the sum of all aperture masks (for correct and incorrect categorisations). This is similar to performing a least-square multiple regression. For each observer, we transformed these probabilities into $Z$-scores, marked with a colour code for the statistically significant $(p<0.05$, one tailed) probabilities, revealing the corresponding features used to perform the gender and expressive or not categorisations (see Fig. 1, Behaviour Classification Image, Accuracy; Chauvin et al., 2005; Gosselin and Schyns, 2001, for details). Reaction time: To determine the features discriminating between fast and slow reaction times on correct trials, we derived a classification image by summing the masks leading to reaction times greater than the mean and those lower than the mean (RTs above and below 2.5 std were treated as outliers and removed from the analysis) and computed the difference. The resulting probabilities were transformed into $Z$ scores, with the statistically significant ( $p<0.025$, two tailed) regions marked with a colour code (not shown in Fig. 1). In Fig. 1, The Behaviour Classification Image (both Accuracy and Reaction Time) mostly represents two eyes, because their presence in the sampled input discriminated correct from incorrect and fast from slow GENDER categorisations.

\section{Computation: Time $\times$ Frequency Classification Images}

To estimate the visual features correlated with Time $\times$ Frequency energy modulations in the signal, on each trial we convolved the measured EEG signal with a complex Morlet wavelet, which has a Gaussian shape in both the time and frequency domain (TallonBaudry and Bertrand, 1999). In this analysis, the centre frequency of the wavelet ranges from $4 \mathrm{~Hz}$ to $30 \mathrm{~Hz}$, in $2 \mathrm{~Hz}$ steps, with a constant ratio of 5 between centre frequency and frequency standard deviation. The time-varying energy of the signal in each frequency band considered is then computed as the square of the modulus of this convolution. To reduce edge effects, the measured signal is 


\section{Stimuli}

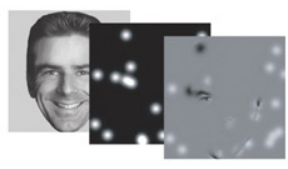

$$
1
$$<smiles>CCCC</smiles>

Time

$x$

Freq

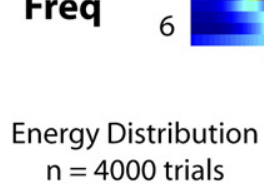

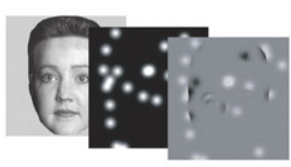

...
4000 trials

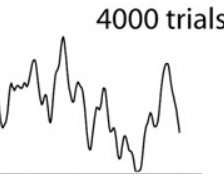

\section{Behavior \\ Classification Image}

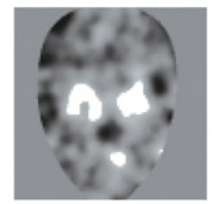

Accuracy

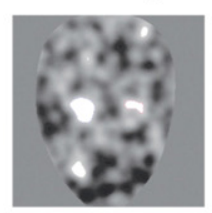

Reaction Time

Time $x$ Frequency

Classification Image

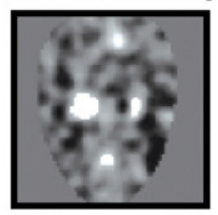

$189 \mathrm{~ms} \times 6 \mathrm{~Hz}$

\section{FTF Map}
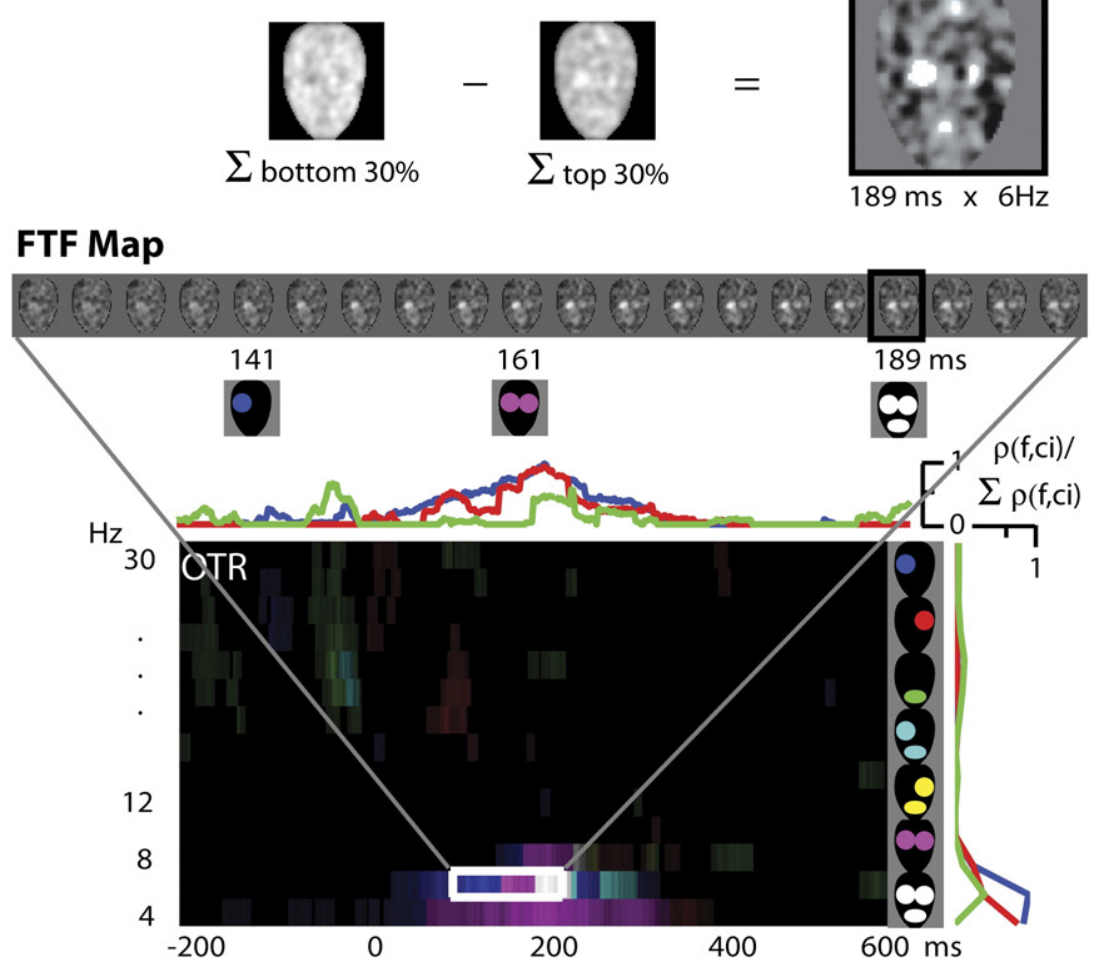

Fig. 1. Bubbles methods applied to EEG measurements (illustrated for observer RZ resolving gender on right occipito-temporal electrode, OTR, P8). Stimuli. Illustration of 2 out of 4000 trials, displaying the original faces, the "bubble masks" that randomly sample facial information on each trial and the resulting facial samples constituting the experimental stimuli. Behaviour Classification Image. Accuracy. In white, significant ( $p<0.05$, one tailed) diagnostic features that this observer used to categorise the gender of the sampled faces, revealing mostly the two eyes. Reaction Time. In white, significant $(p<0.025$, two tailed $)$ diagnostic features that discriminated fast from slow reaction times in correct trials. EEG-Time $\times$ Frequency. On each trial, EEG recorded on face-sensitive OTR electrode P8 between -200 and $600 \mathrm{~ms}$ around stimulus onset is decomposed in a Time $\times$ Frequency $(T \times F)$ analysis (every $2 \mathrm{~Hz}$ from 4 to $30 \mathrm{~Hz}$ ). Across trials, a distribution of 3000-3200 (depending on categorisation performance and artefact rejection) TF energy values exists for each cell of the $T \times F$ matrix (illustrated for $6 \mathrm{~Hz}$ at $189 \mathrm{~ms}$ ). Time $\times$ Frequency Classification Image. We sum the information samples presented on the trials associated with the top (vs. bottom) $30 \%$ of the energy distribution and subtract these sums to derive a classification image representing significant correlations between modulations of facial information and modulations of $T \times F$ energy (illustrated for $6 \mathrm{~Hz}$ at $189 \mathrm{~ms}$, in white, $p<0.001$ ). Feature Time $\times$ Frequency (FTF) Map. This computation repeated for each $T \times F$ cell of the matrix depicts sensitivity of the oscillatory EEG to three basic features (left eye, coded in blue; right eye, in red; mouth, in green). We linearly combine the sensitivities to basic features in RGB space to reveal, with different combinations of colours, combinations of basic features (see Supplementary Fig. 1). To illustrate, the white box in the FTF map highlights a transition, in the 6-Hz band, from 90 to 220 ms after stimulus onset, from processing the contra-lateral eye (coded in blue), followed by two eyes (coded in purple), followed by the two eyes and the mouth, coded in white. RGB line plots collapse the sensitivity measurements, for each basic feature, across time (top plot) and frequency (right plot). 
padded by $200 \mathrm{~ms}$ pre and post the epoch limits. For each point in this Time $\times$ Frequency space (i.e., for each frequency band (4 to $30 \mathrm{~Hz}$ ) and each time point (-200 to $600 \mathrm{~ms})$ ) we derived an independent classification image to determine the features discriminating between low and high EEG energy at this point. To this end, at each point in Time $\times$ Frequency space, we split the distribution of energy values (there is one value per correct non-artifact trial, per time point at each time step, for all frequencies considered) into three bins (the top $30 \%$, middle $40 \%$ and bottom $30 \%$ ). To derive each EEG classification image, we assigned the bubble masks used on each trial to one of the 3 bins as a function of the energy elicited (at each particular time-frequency point) by that bubble mask. This fine split of the EEG time-frequency energy distribution allowed a detailed inspection of how visual information co-varies with EEG Time $\times$ Frequency energy. The Time $\times$ Frequency classification image was the sum of the bubble masks corresponding to the top $30 \%$ of the distribution minus sum of those masks corresponding to the bottom $30 \%$ of the distribution. In Fig. 1, EEG-Time $\times-$ Frequency illustrates this procedure going from raw signal in response to bubbled stimuli to a Time $\times$ Frequency Classification Image. In Fig. 1 , the Time $\times$ Frequency EEG Classification Image represents the two eyes, because their presence in the sampled input systematically modulated OTR oscillatory energy at $189 \mathrm{~ms}$ following stimulus onset, in the $6 \mathrm{~Hz}$ band.

For each observer and categorisation task, repeating this analysis across all frequencies and time points results in a matrix of classification images detailing any facial features that are systematically correlated with the EEG signal in each frequency band and at each time point, for a total of 2 electrodes (OTL and OTR) $\times 2$ tasks $($ EXNEX and GENDER $)=4$ matrices of EEG classification images per observer (see Fig. 1 for an illustration of one vector taken from the matrix).

Relating 1 and 2: The FTF Map: a representation of sensitivity of Time $\times$ Frequency Classification Images to the features of the behaviour classification images

To establish those features that are significantly correlated with the EEG energy at each point in the time-frequency space, we applied a cluster test $(p<0.001$, Chauvin et al., 2005) to each EEG classification image - using the non-diagnostic normalised hairstyle and forehead region as the baseline distribution. Significant regions were then compared (i.e., intersected) with reference templates derived from the diagnostic information of the behaviour classification images (accuracy) of each observer (the left eye, the right eye and the mouth, or $\rho(f, \mathrm{ci})$, for each significant behavioural feature $f$ and Time $\times$ Frequency classification image ci) to extract sensitivity matrices to this information (for a total of 2 electrodes (OTR and OTL) $\times 2$ tasks (GENDER and EXNEX) $\times 3$ basic features $\times 4$ observers $=48$ sensitivity matrices, not shown). ${ }^{2}$ To construct an FTF map, we linearly combine the RGB coefficient of each basic feature (the left eye, coded in blue, the right eye, coded in red, and

\footnotetext{
${ }^{2}$ It is important to note that we computed an intersection between each basic feature and a Time $\times$ Frequency classification image, not a correlation. The intersection allows the basic feature templates to be used as an orthogonal space to project the Time $\times$ Frequency classification image - i.e., the intersection between the feature templates is an empty set. An alternative measure such as a correlation between each basic feature and the Time $\times$ Frequency classification image does not produce one such orthogonal space because the feature templates are not orthogonal to one another and do not form a basis. Instead, they are negatively correlated.
}

the mouth, coded in green, represented in independent sensitivity matrices). This linear combination produces colour-coded feature combinations, when the classification image intersects with several features (i.e., the two eyes in purple, the left eye and the mouth in turquoise, the right eye and the mouth in yellow and the left eye, the right eye and the mouth in white, see Supplementary Fig. 1). The linear combination is warranted because the three basic features (left eye, right eye and mouth) constitute, for each subject, an orthogonal basis onto which the Time $\times$ Frequency classification images can be projected. Linearly adding the three sensitivity matrices produces one FTF map per combination of observer, task and electrode (e.g., RZ resolving GENDER on OTR in Fig. 1). The FTF map establishes the sensitivity to individual features (e.g., the left eye, coded in blue) or combinations of features (e.g., the two eyes, coded in purple) and the time course of transitions between different information processing states. To illustrate, in Fig. 1, on OTR, in the $6 \mathrm{~Hz}$ band, the presence of the contra-lateral left eye at $141 \mathrm{~ms}$ is coded in blue, followed by the presence of two eyes at $161 \mathrm{~ms}$ (coded in purple) and the presence of the three features at $189 \mathrm{~ms}$ (coded in white). The FTF map is a novel solution to the foundational problem of inferring the information processing states and transitions of a cognitive mechanism between stimulus onset and response, and thereby relating a cognitive mechanism implemented in the brain to behaviour.

At this stage, it is worthwhile pointing out the critical difference between this approach and other more typical approaches (see Schyns et al., 2003; Smith et al., 2004, 2005, 2006; Nielsen and Rainer, 2007; Gosselin and Schyns, 2001 for discussions). Most brain imaging studies of visual categorisation aim to establish "that category $X$ elicits a significantly different brain response than control categories $Y, Z$ and so forth." The shortcoming of this approach is that it leaves the reader guessing the precise visual information associated with the significant response difference. Our approach addresses this shortcoming by establishing a precise mapping between the variance of a brain response and input information, and the variance of a behavioural response and input information. Of course, one could always select a priori a number of features and test them against brain responses. However, this does not establish a precise mapping between the variance of the input information and the variance of the brain responses (see, e.g., Smith et al., 2004, for precise depictions of this correspondence over the N170 and the P300 ERPs). Furthermore, there is always the unresolved problem of the experimenter bias in choosing which information to test, which information to control, and which information to disregard. Here, we are testing all of the available information, by sampling it randomly across trials. The FTF maps and the behaviour classification images therefore represent different regressions of the same set of information samples with different responses. Critically, it is the different responses (i.e., OTL and OTR electrodes and categorisation accuracy) that are sensitive to different face information, leading to different classification images from the same input information samples. The strength of this approach results from its ability to compare behavioural and brain measurements within the same space (the input stimulus) to better understand functionality.

\section{Results}

\section{Behavioural information}

MB was correct on $92 \%$ of the trials in GENDER (RT: $\mu=$ $861 \mathrm{~ms} ; \sigma=271 \mathrm{~ms}$ ) and $90 \%$ in $\operatorname{EXNEX~(RT:~} \mu=900 \mathrm{~ms} ; \sigma=$ 
$330 \mathrm{~ms}$ ); RZ 85\% (RT: $\mu=709 \mathrm{~ms} ; \sigma=192 \mathrm{~ms}$ ) and 90\% (RT: $\mu=732 \mathrm{~ms} ; \sigma=237 \mathrm{~ms}$ ); ML $88 \%$ (RT: $\mu=790 \mathrm{~ms} ; \sigma=210 \mathrm{~ms}$ ) and $87 \%$ (RT: $\mu=730 \mathrm{~ms} ; \sigma=185 \mathrm{~ms}$ ); BB $81 \%$ (RT: $\mu=846 \mathrm{~ms}$; $\sigma=168 \mathrm{~ms}$ ) and $80 \%$ (RT: $\mu=760 \mathrm{~ms} ; \sigma=152 \mathrm{~ms}$ ), respectively. The classification image analysis revealed that the accuracy of observers mostly depended on the presence of two eyes and some of the mouth in GENDER and on the exclusive presence of the mouth in EXNEX (henceforth, we consider a feature to be a cluster of connected classification image pixels above a statistical threshold; here, $p<0.05$ ). A similar use of information discriminated fast from slow reaction times (see Figs. 2, 3 and Supplementary Figs. 2 and 3, Behaviour Classification Image). It is important to stress that these features constitute the minimal face information that the brain must process in order to resolve the two face categorisations. Thus, we would expect modulations of the EEG oscillatory energy to reflect at least this information content between stimulus onset and behavioural response.

\section{FTF maps}

To reveal the information content associated with modulations of oscillatory energy, we computed independent FTF maps for each observer, electrode and categorisation task. For each cell of a Time $\times$ Frequency analysis applied to the EEG recorded on each correct trial of a task, we computed a Time $\times$ Frequency classification image to establish the facial features systematically correlated with variations of energy. We then classified the Time $\times$ Frequency classification image as to the degree of presence of the left eye (in blue), the right eye (in red) and the mouth (in green). The colour coding of features is purposeful: linear combinations of colours will reflect the combinations of two or more basic features in the classification image (e.g., the left eye and the mouth: blue + green in RGB coding = turquoise). Repeating the approach to each Time $\times$ Frequency cell produces an FTF map from which we will infer distinct information processing states and their transitions (see Methods and Fig. 1 and Supplementary Fig. 1).

\section{Information Processing States}

Consider the FTF map of observer BB resolving GENDER (Fig. 2A). We know from the behavioural analysis that this observer uses mostly the two eyes to categorise the faces as male and female (see Behaviour Classification image, Accuracy and Reaction Time in Fig. 2A). On an OTR electrode, in a band
A
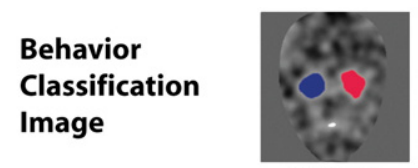

Accuracy

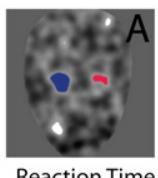

OTR
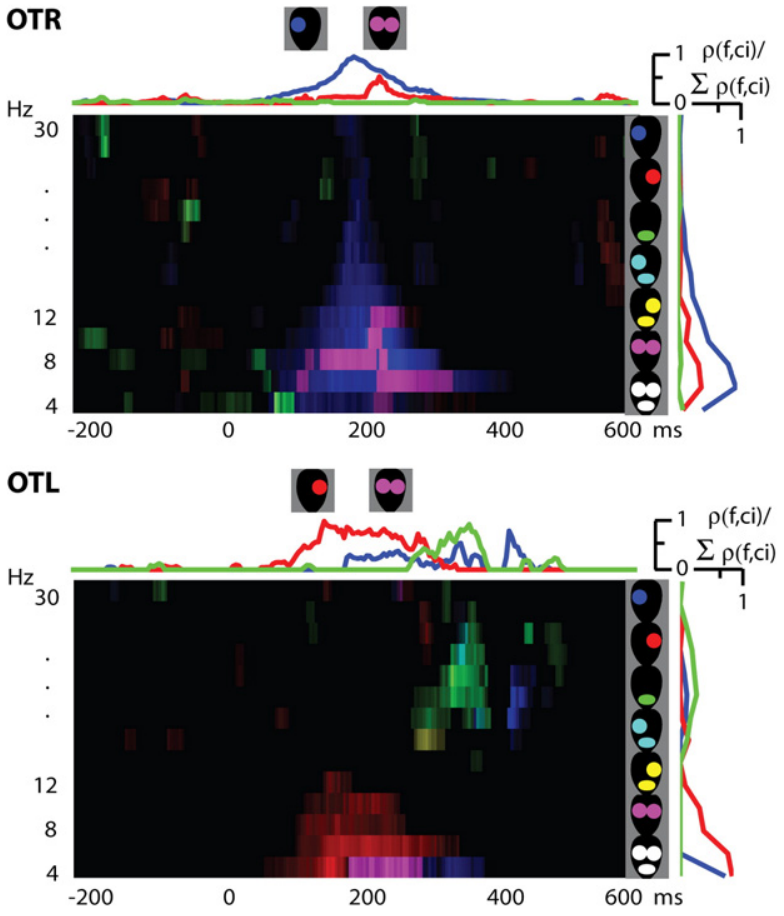

B
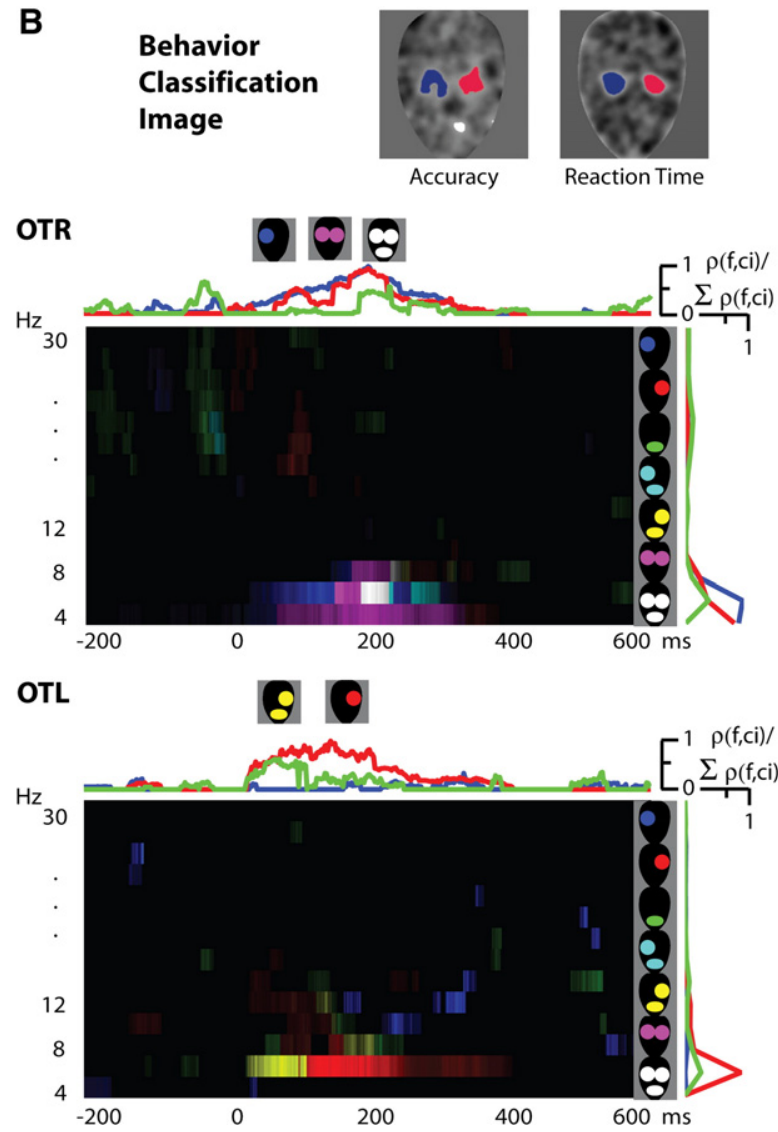

Fig. 2. (A, Left) Feature Time Frequency Maps: BB resolving GENDER. Behaviour Classification Image. Accuracy. In blue and red, significant ( $p<0.05$ one tailed) diagnostic features that BB used to categorise the gender of the sampled faces, revealing mostly the two eyes. Reaction Time. Using the same colour coding, significant ( $p<0.025$ two tailed) diagnostic features that discriminated fast from slow reaction times in correct trials. OTR and OTL Feature Time Frequency Maps. On OTR, between 100 and $300 \mathrm{~ms}$, and between 4 and $12 \mathrm{~Hz}$, a state of sensitivity to the contra-lateral left eye (coded in blue) is present before a state of sensitivity to both eyes (coded in purple). On OTL, in the same Time $\times$ Frequency window, sensitivity to the contra-lateral right eye (coded in red) is also present before sensitivity to both eyes (coded in purple). RGB line plots. Collapsing the sensitivity measurements, for each basic feature, across time (top plot) and frequency (right plot). On OTR and OTL, the top plots illustrate the precedence of the contra-lateral eye over the ipsi-lateral eye between 100 and 300 ms. The schematic, colour-coded facial features illustrate the corresponding state transitions. The right plot illustrates that feature sensitivity encompasses theta and alpha EEG oscillations-i.e., 4 to14 Hz. (B, Right). Feature Time Frequency Maps: RZ resolving GENDER. 
A
Behavior Classification Image

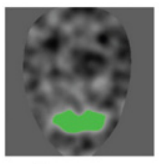

Accuracy

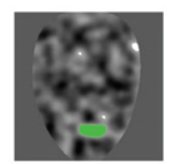

Reaction Time
B
Behavior Classification Image

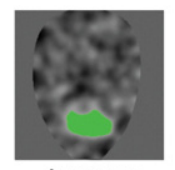

Accuracy

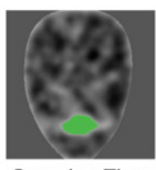

Reaction Time
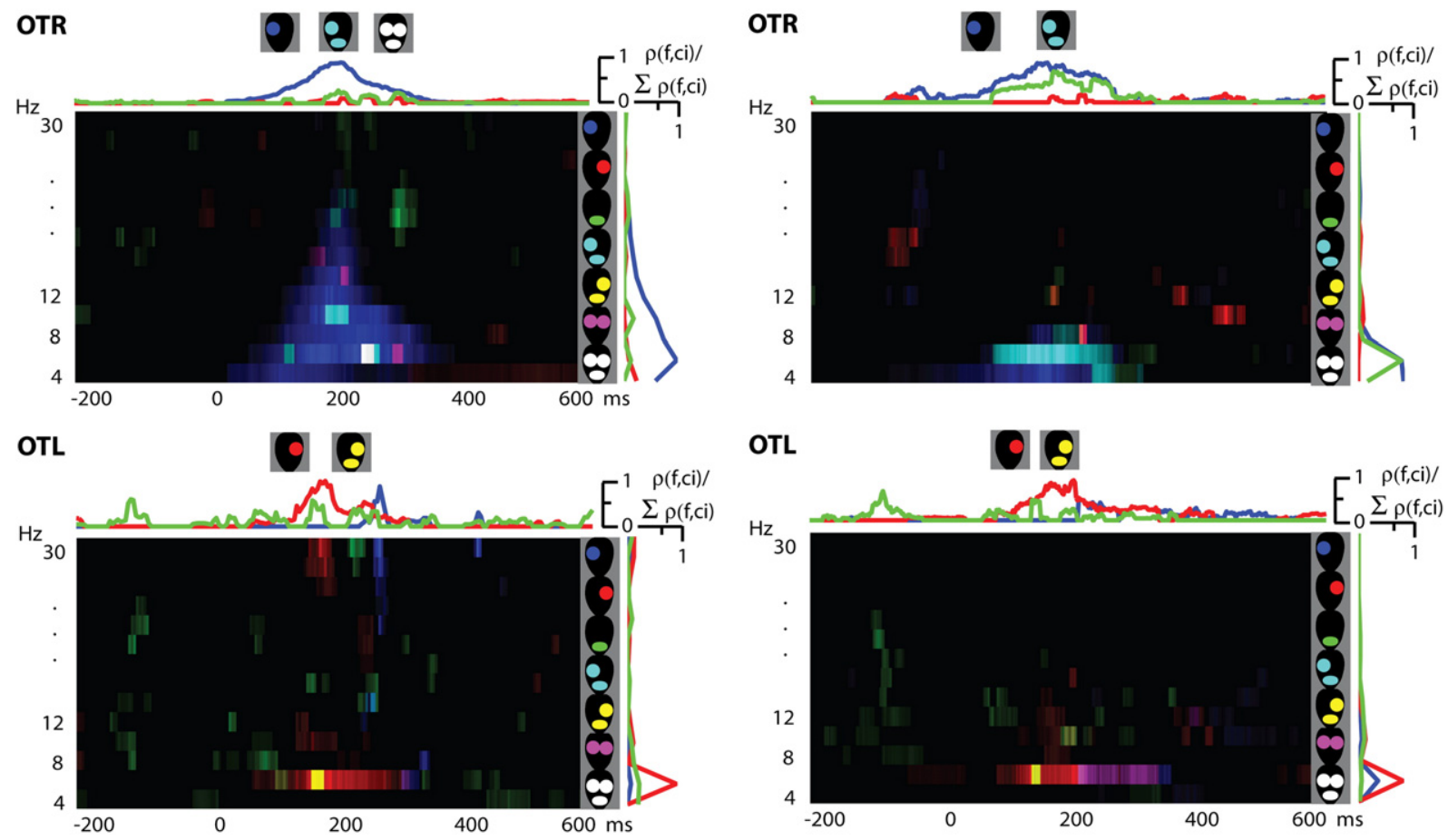

Fig. 3. (A, Left) Feature Time Frequency Maps: BB resolving EXNEX. Behaviour Classification Image. Accuracy. In green, significant ( $p<0.05$, one tailed) diagnostic features that $\mathrm{BB}$ used to categorise the expression of the sampled faces, revealing mostly the mouth. Reaction Time. Using the same colour coding, significant $(p<0.025$, two tailed) diagnostic features that discriminated fast from slow reaction times in correct trials. OTR and OTL Feature Time Frequency Maps. On OTR and OTL, between 100 and $300 \mathrm{~ms}$, and between 4 and $12 \mathrm{~Hz}$, a state of sensitivity to the contra-lateral left eye (coded in blue) is present before a state of sensitivity to the contra-lateral eye and the mouth (coded in both turquoise on OTR and in yellow on OTR). Information processing states therefore start with the contra-lateral eye, to evolve to the eye and the mouth and finish with the mouth, as predicted by the Behaviour Classification Image. RGB line plots. On OTR and OTL, the top plots illustrate the interplay between initially contra-lateral eye then eye and mouth information. The right plot illustrates that feature sensitivity encompasses theta and alpha EEG oscillations-i.e., 4 to14 Hz. (B, Right) Feature Time Frequency Maps: RZ resolving EXNEX.

extending from 4 to $16 \mathrm{~Hz}$ and in a time window extending from around 100 to $300 \mathrm{~ms}$ following stimulus onset, there is a clear sensitivity to the contra-lateral left eye (coded in blue in Fig. 2A, OTR FTF map). On the opposite OTL electrode, in the same frequency and time window, sensitivity to the contra-lateral right eye is apparent (coded in red in Fig. 2A, OTL FTF map). Thus, observer BB processes the left and right eyes to resolve GENDER, but contra-laterally, in different hemispheres, resulting in two distinct states of information processing. Sensitivity to the conjunction of the left (blue) and right (red) eye (coded in purple in Fig. 2A) is an information state present both on OTR and OTL, within the same overall time window as sensitivity to individual eyes, but slightly lower in frequency (i.e., between 4 to $12 \mathrm{~Hz}$ ), as predicted by the fact that categorisation accuracy is based on the presence of this content (the two eyes) in the input (see Fig. 2A, Behaviour Classification Image).

Turning to the same observer resolving EXNEX, the OTR and OTL maps in Fig. 3A reveal sensitivity to individual contra-lateral eyes (the blue and red colours representing the left and right eyes respectively), but more pointedly, sensitivity to the contra-lateral eye and the mouth (represented in turquoise vs. yellow on OTR vs. OTL in Fig. 3A). These conjunctions are both represented in the 6-Hz band, around $200 \mathrm{~ms}$ following stimulus onset. Finally, we identify an information state involving all the individual features under scrutiny: the left (blue) and right (red) eye and the (green) mouth. This state, present on electrode OTR in the 6-Hz frequency band, at $220 \mathrm{~ms}$, extends for approximately $20 \mathrm{~ms}$ (represented in white in Fig. 3A). In EXNEX, categorisation accuracy depends on the presence of the mouth in the input a content processed on both electrodes.

A similar pattern of results in the two tasks is present for observer RZ (see Figs. 2B and 3B). Note in particular the $6-\mathrm{Hz}$ sensitivity to the contra-lateral eye and the mouth in EXNEX (represented in turquoise vs. yellow on OTR vs. OTL in Fig. 3B) and note also the sensitivity to both eyes in GENDER (represented in purple in Fig. 2B). Observers ML and MB were also similar to each other in their information processing states, though theirs included more feature conjunctions (encoded in purple and in white in Supplementary Figs. 2A, B and 3A, B).

Thus, the FTF maps on face processing sensitive OTR and OTL electrodes reveal distinct, identifiable information processing states between stimulus onset and behavioural response, in the GENDER and EXNEX face categorisations. These information states reflect the processing of individual features (the left or right contra-lateral eye, or the mouth) or conjunctions of features (the two eyes, the left 
or right contra-lateral eye and the mouth). It is important to understand that our experiment is a three-factor ( 2 electrodes $\times 200$ time points $\times 15$ frequency bands) exhaustive within-observer design. Each cell of the FTF map represents its own selective sensitivity (if any) to the same uniform distribution of input information and serves as control to the other cells. Identifiable information states (e.g., the left eye on OTR and the right eye on OTL, the mouth and feature combinations) illustrate intricate interactions between the three factors.

\section{State Transitions and Time Course}

The FTF maps also embed the time course and transitions of information processing states. RGB line plots on top of each FTF map in Figs. 2 and 3 collapse the frequency dimension to represent the time course of sensitivity to the basic left eye (blue), right eye (red) and mouth (green). Overlap between basic features reveals conjunctive sensitivities. Retuning to observer $\mathrm{BB}$ resolving GENDER in Fig. 2A, OTR electrode reveals an initial processing of the contra-lateral left eye (in blue), followed by the conjunction of the contra-lateral and ipsi-lateral eyes (in purple). A similar state transition appears on electrode OTR, starting this time with the contra-lateral right eye (in red) followed by both eyes (in purple).

For BB resolving EXNEX in Fig. 3A, OTR and OTL electrodes also reveal state transitions involving initially the contra-lateral eye (i.e., the left eye in blue for OTR and the right eye in red for OTL), followed here by a conjunction of the left eye and the mouth
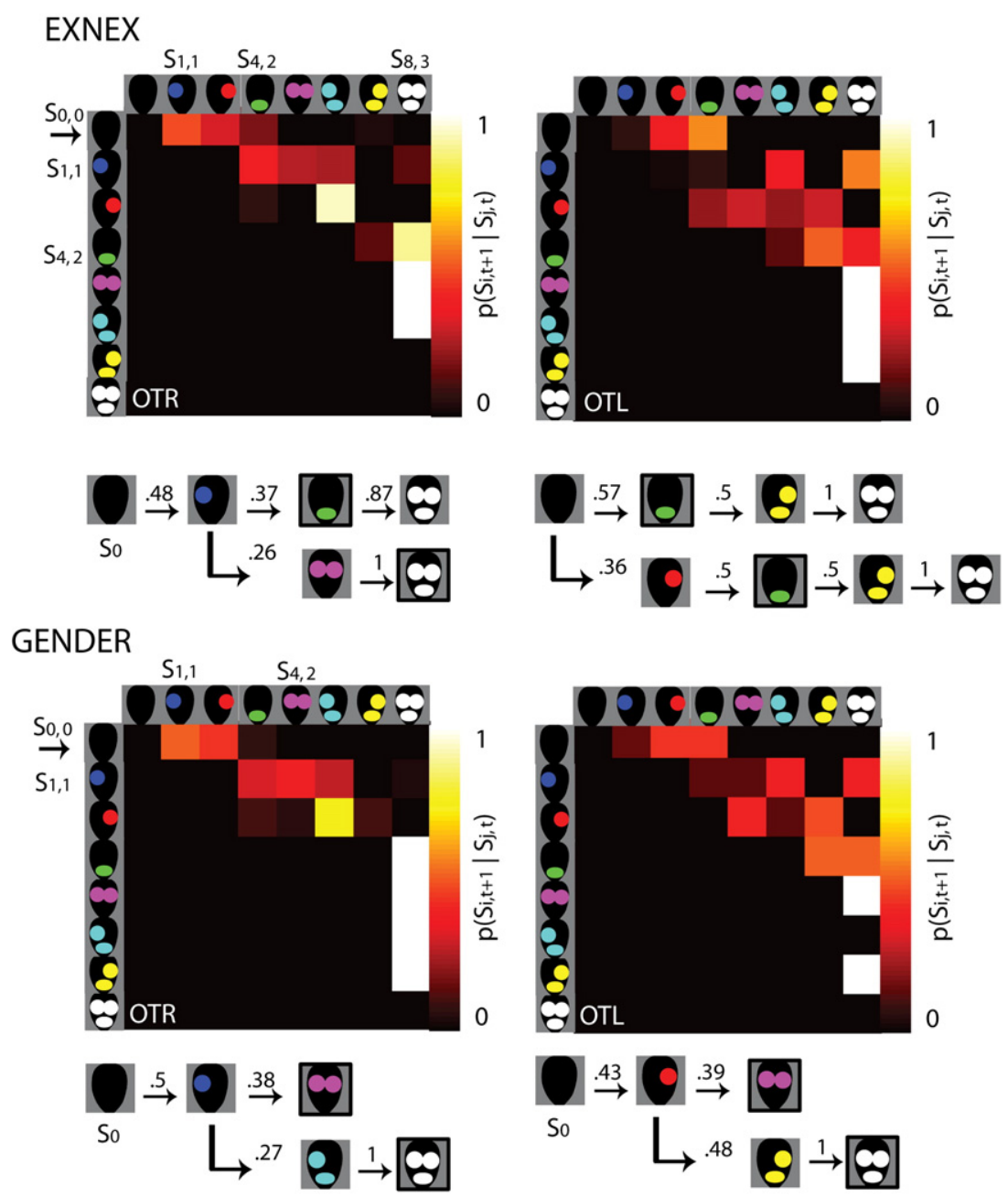

Fig. 4. (A, Top). Stochastic automata: EXNEX. For electrode OTR and OTL, a table specifies the conditional probabilities of transitions between states $p\left(S_{i, t+1} \mid S_{j, t}\right)$. Underneath the tables, two strings of states illustrate the two most likely chains of transitions for each electrode. To derive one transition table per electrode, we collapsed the EXNEX FTF maps across observers, in a Time $\times$ Frequency window lasting between 60 and 350 ms following stimulus onset and spanning 4 to $14 \mathrm{~Hz}$ (where most information states are located). We computed, without thresholding, only using the linear combinations of the three basic features, all possible transition probabilities between state $S_{i}$ and $S_{j}$, excluding self-similar transitions (i.e., $S_{i} \rightarrow S_{i}$ ) and all transitions between a high state of information (e.g., all features) and a low state of information (e.g., no information, or the two eyes, or one eye). This resulted in one diagonal transition table per electrode. To read these tables properly, start in the left column with state $S_{0}$, at time 0 ( $S_{0,0}$, indicated with an arrow). In the adjacent row, a colour codes the conditional probability of transition to $S_{i}$ (colour-coded in the tow row) at time $1\left(S_{i}, 1\right)$, with $\sum_{i=1 \ldots 8} p\left(S_{i, 1} \mid S_{0,0}\right)=1$. The most likely transition is $S_{0,0} \rightarrow S_{1,1}$, the contra-lateral left eye, coded in blue. Reporting $S_{1,1}$ in the left column of states, the process can be repeated to find that the most likely transition is $S_{4}$ at time 2 $\left(S_{4,2}\right)$, the mouth. Halting states are those for which there is no transition to another state. These show that the diagnostic information for behaviour for the EXNEX categorisation (i.e., the mouth) is reached within a few information processing state transitions, independently on OTR and OTL. (B, Bottom). Stochastic automata: GENDER. The automata show that the diagnostic information for behaviour for the GENDER categorisation (i.e., the two eyes) is reached within a few information processing state transitions, independently on OTR and OTL. 
(turquoise) then the two eyes and the mouth (white) on OTR and by the right eye and the mouth (yellow) on OTL.

A similar pattern of state transitions characterises RZ for GENDER and EXNEX (see Figs. 2B and 3B). For ML and MB, state transitions reflect the higher level of information represented in each state, evolving from the conjunction of two features (mostly the two eyes encoded in purple in Supplementary Figs. 2, 3) to the conjunction of three features (encoded in white). Though not the most parsimonious strategy, they encoded all the information required to resolve the two categorisations.

Despite an obvious difference in information quantity between $\mathrm{BB}, \mathrm{RZ}$ and $\mathrm{ML}, \mathrm{MB}$, all four observers displayed similar state transition dynamics, starting with contra-lateral eye information on OTR and OTL, followed by conjunctive information (but more of it, sooner in ML and MB). State transitions therefore evolved towards an increase in information complexity, on both occipito-temporal electrodes, between stimulus onset and response.

\section{Time Course and Stochastic Automata}

The characterised information states are a mixture of information directly relevant for behaviour (i.e., diagnostic, such as the mouth in EXNEX) and other features (e.g., the eyes in EXNEX). As discussed, diagnostic features are particularly important because the observer must process them in order to properly categorise the faces. Thus, evidence of diagnostic features in the information state transitions is an important marker of a neural correlate of behaviour. To characterise the time course of diagnostic information processing, we collapsed observers per categorisation task (GENDER and EXNEX) and electrode (OTR and OTL) and isolated the Time $\times$ Frequency window within which the intersection of the diagnostic features (two eyes in GENDER, the mouth in EXNEX) with Time $\times$ Frequency classification image reached threshold. The white boxes in Supplementary Figs. 4A and B highlight a 6- to 8-Hz sensitivity to diagnostic features on OTR and OTL in GENDER and EXNEX, always preceded by sensitivity to the contra-lateral eye information. There is a marginal time advantage for the onset of two eyes in GENDER on OTR (44ms gain) and OTL (24-ms gain, compare Supplementary Figs. 4A and B) over the mouth in EXNEX (see Supplementary Fig. 5B) that is not reflected in reaction time differences across tasks (GENDER: $\mu=802 \mathrm{~ms} ; \quad \sigma=210 \mathrm{~s}$; EXNEX: $\mu=781 \mathrm{~ms}$; $\sigma=226 \mathrm{~ms}$, ns).

Finally, coming back to our aim of modeling each categorisation from brain signals in terms of a finite state automaton, we provide probabilistic transition tables (computed across all observers and temporal frequencies) for each categorisation task and electrode. First, we set up the finite set of states by building an exhaustive list of all the states present across the four observers. Then, we compute, across the four observers, the conditional probabilities of transitions from state $i$ at time $t, S_{i, t}$, to state $j$ at time $t+1, S_{j, t+1}$. To illustrate for EXNEX and electrode OTR, consider the left transition table in Fig. 4A. Starting at time 0 with a state of no information processing $S_{0,0}$ (see arrow in Fig. 4A), the first row describes the conditional probabilities of a transition to any other possible state $p\left(S_{i}={ }_{1 \ldots, 8,1} \mid S_{0,0}\right)$. The most likely transition at time $=1$ is $S_{1}$, the contra-lateral left eye (encoded in blue), with a 0.48 conditional probability of transition. From $S_{1,1}$ in the left column, the most likely conditional transition at time 2 is $S_{4}$, the mouth (encoded in green), with a 0.37 probability (the surrounding black box indicates that the state comprised diagnostic information). From $S_{4,2}$ at time 2 , the most likely transition at time 3 is $S_{8}$ (encoding all three features), with a probability of 1 . The two most likely chains of transitions are reported at the bottom of the transition tables in Fig. 4A. For a given categorisation, the comparison between OTR and OTL highlights the higher likelihood of an initial encoding of the contra-lateral eye (compared with the ipsi-lateral eye). Across categorisations (compare Figs. 4A and B), diagnostic information is reached within two transitions on OTR; it is reached between 1 and 3 transitions on OTL. Note also the progression of information states: the transition to a feature combination (e.g., one eye and the mouth) can only be reached from one of the component features, never from $S_{0,0}$. Similarly, the probability of a transition from $S_{0,0}$ to $S_{8}$ (all three features) is 0 . Together, Figs. $4 \mathrm{~A}$ and B detail the categorisation-specific sequences of information processing states from the occipito-temporal regions linking the input face to a specific categorisation response.

Finally, Fig. 5 presents the most likely state transitions in each categorisation task. The left panel reveals significant transitions ( $p<0.05$ confidence interval estimated for a Bernoulli 0.5 distribution of transitions to tasks) from the contra-lateral eyes to the two eyes in GENDER (see corresponding letter $G$ in the figure) whereas state transitions were more to the mouth in EXNEX (see corresponding letter $\mathrm{E}$ in the figure). This frequency of significant transitions is reported in the right histogram, where the two eyes and the mouth stand for transitions to this information.

\section{Discussion}

Individual observer's FTF maps and the finite state automata have suggested (1) distinct visual information processing states and (2) transitions between states over the time course of two face categorisation tasks. States and their transitions were identified mostly in the low-frequency EEG, in the theta [4-8 Hz] and alpha $[8-12 \mathrm{~Hz}]$ bands. The results suggest a time line of distinct feature

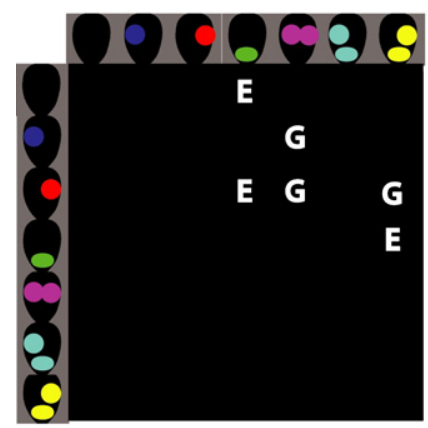

State Transitions Differences

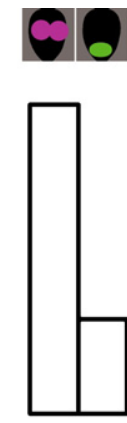

GENDER

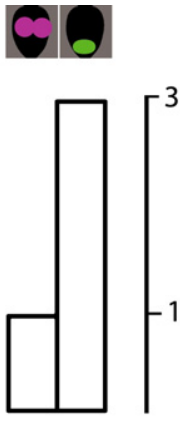

EXNEX
Fig. 5. State transition differences between GENDER and EXNEX. This figure illustrates the significant differences $(p<0.05$, confidence interval estimated for a Bernoulli 0.5 distribution of transitions to tasks) between tasks, in frequency feature state transitions (collapsed across observers, electrodes and temporal frequencies). A letter standing for the task $(\mathrm{E}=\mathrm{EXNEX} ; \mathrm{G}=\mathrm{GENDER})$ reveals the task for which the state transition difference was significant. Examining the nature of the transitions involved in GENDER and EXNEX, it is clear that GENDER involved more transitions from one of the eyes to the two eyes (the diagnostic information) whereas EXNEX involved more transitions to the mouth (the diagnostic information). Note that the state embedding all three features was removed from this analysis, because it is essentially ambiguous with respect to tasks. The histogram to the right indicates, for each task, state transitions involving eye (left, or right, or both) vs. mouth information in the two tasks. 
processing states in the brain on OTR and OTL electrodes, revealing for the first time the dynamics and specificity of cognitive face processing mechanisms in different face categorisation tasks. Following stimulus onset, a state of processing to the contra-lateral eye initiates the mechanism on OTR and OTL, irrespective of categorisation task. Following this apparently automatic encoding of contra-lateral eye information, processing states change to become specific to task-dependent, bilateral information. Specifically, later processing epochs on OTR and OTL were more sensitive to both eyes than the early epochs in GENDER and to the mouth and the eyes in EXNEX.

It is surprising that eye information is processed by all observers (and on both electrodes) before the mouth when arguments of efficiency and the blocked nature of the design should bias observers to extract the mouth first in EXNEX. A number of reasons could explain this sequence: the eyes are important for many natural face computations (including judgments of emotions; Adams et al., 2003; Adolphs et al., 2005; Smith et al., 2005; identity, Bentin et al., 1996; Gosselin and Schyns, 2001; Schyns et al., 2002; and gaze, Schuller and Rossion, 2001) and so their initial encoding could lead to more efficient categorisations of faces in general, given the prevalence of this information across tasks (Gosselin and Schyns, 2001; Smith et al., 2005; Schyns et al., 2002). Another reason is that the eyes provide a useful computational landmark to locate other features and so are used as reference from which diagnostic features can be extracted and added to a dynamic representation of the input for categorisation.

\section{Functional interpretation of information state transitions}

Our approach should enable a finer functional interpretation of the role of the brain structures underlying OTR and OTL activity. However, we must be cautious and point out that this functional interpretation is problematic with EEG measurement, given its low spatial resolution. Still, we know that the transition of information states from contra-lateral to diagnostic information on occipitotemporal cortex ensures that the underlying brain processes are involved with the information of the face categorisation tasks. An interpretation of this involvement is that we are observing the brain in action, progressively forging featural face representations for different categorisations, starting with the eyes, contra-laterally, then adding bilateral diagnostic information. The initial contralateral nature of the process suggests a relative independence of the hemispheres early on, until evidence of bilateral information suggests that an inter-hemispheric communication makes the information of each hemi-field available across hemispheres. When diagnostic information is processed (between 150 and $250 \mathrm{~ms}$ ), visual categorisation systems have computed the information required for categorisation decision and motor behaviour. This would extend findings that inferior temporal cortex neurons in nonhuman primates are sensitive to diagnostic object properties (Freedman et al., 2003; Logothetis et al., 1995; Nielsen et al., 2006; Sigala and Logothetis, 2002) by providing, with a more integrated neural signal, the state transitions leading to the diagnostic representation.

One could object that we are not observing the constructions of these representations per se, but rather the correlates of one (or several) of the processes logically afferent to the construction of representations, such as attention to, or feature encoding, or even eye movements. Eye movements are easily ruled out because EEG signals with eye movement artifacts were excluded from the analyses. Furthermore, eye movement research (e.g., Rayner, 1998) has shown that in picture viewing, a fixation lasts for about 300 to $400 \mathrm{~ms}$. If a saccade lasts for about $50 \mathrm{~ms}$ (though this depends on length of saccade), the rate of saccadic eye movements is about $3 \mathrm{~Hz}$ $(1000 / 350 \mathrm{~ms})$ to $2.5 \mathrm{~Hz}(1000 / 400 \mathrm{~ms})$. The rate of saccadic eye movements is therefore well below the rate of the reported state transitions. Finally, and crucially, the evidence of a sequential accumulation of features over a short time window (e.g., the left eye, the left eye and the right eye, the left eye and the right eye and the mouth, over $100 \mathrm{~ms}$ on OTR) implies a process that integrates features and represents this integration over time (i.e., has a memory), not a process that sequentially visits independent features, as saccadic eye movements would.

In the context of this feature integration, theta and alpha EEG oscillatory bands act as "carrier" for the information processing states and their transitions. Alpha-band EEG oscillations have been related to task demands, task load and increasing demands of attention (Foxe et al., 1998; Fu et al., 2001; Klimesch, 1999) both in the auditory (Large and Jones, 1999) and the visual (Varela et al., 1981; see also Gambini et al., 2002; Gho and Varela, 1988) domains. Our reported feature processing in the theta to alpha oscillatory band is suggestive of increased attention to features in both categorisation tasks, particularly early on in face processing, when faces must be detected in the stimulus.

On the other hand, theta oscillations have also been associated with the encoding of information in memory tasks (Axmacher et al., 2006; Basar et al., 2000; Buzsaki, 2002; Klimesch, 1999; Pare et al., 2002). In the model of Lisman and Idiart (1995), it is proposed that slow brain rhythms (e.g., theta and alpha) mediate the construction of discrete representations, the content of which would be fleshed out by faster rhythms (see also Ritz and Sejnowski, 1997). Such interactions between slow and fast brain rhythms have been proposed to reflect brain mechanisms that are global (to link distinct brain regions), quasi-periodic (i.e., with an active and an inactive phase) and flexibly adjustable (to adapt their period and amplitudes to constraints, both internal and external, Van Rullen and Koch, 2003). The evidence of an integration of facial features over time in the theta carrier reported here is compatible with the idea that facial information is progressively built over transitions to forge flexible, task-dependent representations for face categorisations.

At this stage, however, we cannot fully tease apart a "representation" from an "attention" account of the information states and it is not clear that such distinction is useful (Schyns, 1998). The existing literature proposes that theta and alpha rhythms can be related to each account and it is still a matter of considerable debate how the two accounts can be separated (e.g., Van Rullen and Koch, 2003; Ward, 2003). To illustrate the ensuing challenge for visual cognition, one could assume that the first information processing state to the contra-lateral eye reflects attention to features early on in the recognition process. But then, this attended information would need to be encoded and rehearsed in visual memory while other features (e.g., the other eye and/or the mouth) are also attended and added to the constructed representation until diagnostic features information is integrated. In this putative process, attention, memory and representation are intertwined functions. We know that the reported information states evolve from contra-lateral to bilateral and from apparently automatic (to the eyes) to more strategic (to diagnostic information) between stimulus onset and response. However, only further theoretical developments and extensive research would tease apart the information states that 
are related to this (e.g., attentional) or that stage (e.g., representational, memory) of the recognition process.

\section{Relationship to EEG and face processing}

There is a vast literature on the EEG and face processing, which has revealed that faces (Bentin et al., 1996; Eimer, 2000; Rossion et al., 2000; Rossion et al., 2003), the encoding of facial structure (de Haan et al., 1998; Rossion et al., 1999, Sagiv and Bentin, 2001), the eyes (Bentin et al., 1996; Schyns et al., 2003; Smith et al., 2004), facial emotion (Batty and Taylor, 2003; de Haan et al., 1998; Eimer and Holmes, 2002) subordinate objects and expert categories (Rossion et al., 2002) modulated the average EEG (the event related potential, or ERP) at a latency of $170 \mathrm{~ms}$ following stimulus onset, though a recent controversy has attributed these effects to low-level stimulus properties (Thierry et al., 2007). Our methods allow a clear falsification of the latter position: the low temporal frequencies of the EEG (which would represent the ERP) represented the contralateral eye, a specific facial feature, not uncontrolled variance of low-level properties. Sensitivity to the contra-lateral eye is reflected in some proposals (Bentin et al., 1996; Smith et al., 2004) regarding one possible function of the N170. It is interesting to note, however, that processing on OTR and OTL electrodes does not end with the N170 ERP. In fact, sensitivity to information continues and accrues new feature over time until about $260 \mathrm{~ms}$ following stimulus onset. Schweinberger et al. (2002) reported that the repetition of familiar items elicited an N250 component, but at slightly more temporal locations, possibly reflecting different sources. Such tracking of information flow across sources should be the object of future studies.

\section{Conclusions}

We have related brain activity to the states of a cognitive information processing mechanism. In doing so, we have addressed three questions: to address the question of the brain activity supporting face processing, we examined a time-resolved brain measurement (Time $\times$ Frequency oscillatory energy) on facesensitive electrodes. To address the question of the information content processed in the oscillatory energy, we applied a novel classification image to produce the FTF maps and identified distinct information processing states. We have used the FTF maps to address the question of how information flows between stimulus onset and behavioural response and proposed a different mechanism for two face categorisation tasks that integrates information over different states. We believe that the approach outlined here applied to brain measurements (EEG, MEG, fMRI and single cell recording) could offer considerable insight into the response properties of interactive brain signals (Eckhorn et al., 1988; Engel et al., 1991; Fries et al., 2001; Gray et al., 1989; Singer, 1999).

\section{Acknowledgment}

This research was supported by an Economic and Social Research Council grant (R000237901) awarded to P.G.S.

\section{Appendix A. Supplementary data}

Supplementary data associated with this article can be found, in the online version, at doi:10.1016/j.neuroimage.2007.05.030.

\section{References}

Adams Jr., R.B., Gordon, H.L., Baird, A.A., Ambady, N., Kleck, R.E., 2003. Effects of gaze on amygdala sensitivity to anger and fear faces. Science $300,1536$.

Adolphs, R., Gosselin, F., Buchanan, T.W., Tranel, D., Schyns, P., Damasio, A.R., 2005. A mechanism for impaired fear recognition after amygdala damage. Nature 433, 68-72.

Axmacher, N., Mormann, F., Fernandez, G., Elger, C.E., Fell, J., 2006. Memory formation by neuronal synchronization. Brain Res. Rev. 52, $170-182$.

Basar, E., Basar-Eroglu, C., Karakas, S., Schurmann, M., 2000. Brain oscillations in perception and memory. Int. J. Psychophys. 35, 95-124.

Batty, M., Taylor, M.J., 2003. Early processing of the six basic facial emotional expressions. Cogn. Brain Res. 17, 613-620.

Bentin, S., Allison, T., Puce, A., Perez, A., McCarthy, G., 1996. Electrophysiological studies of face perception in humans. J. Cogn. Neurosci. 8, 551-565.

Buzsaki, G., 2002. Theta oscillations in the hippocampus. Neuron 3, $325-340$.

Chauvin, A., Worsley, K.J., Schyns, P.G., Arguin, M., Gosselin, F., 2005. Accurate statistical tests for smooth classification images. J. Vis. 5, 659-667.

De Hann, M., Nelson, C.A., Gunnar, M.R., Tout, K.A., 1998. Hemispheric differences in brain activity related to the recognition of emotional expressions by 5 year-old children. Dev. Neuropsychol. 14, 495-518.

Eckhorn, R., Bauer, R., Jordan, W., Brosch, M., Kruse, W., Munk, M., Reitboeck, H.J., 1988. Coherent oscillations: a mechanism of feature linking in the visual cortex? Multiple electrode and correlation analyses in the cat. Biol. Cybern. 60, 121-130.

Eimer, M., 2000. Effects of face inversion on the structural encoding and recognition of faces-Evidence from event-related potentials. Cogn. Brain Res. 10, 145-158.

Eimer, M., Holmes, A., 2002. An ERP study on the time course of emotional face processing. NeuroReport 13, 427-431.

Engel, A.K., Kreiter, A.K., Konig, P., Singer, W., 1991. Synchronization of oscillatory neuronal responses between striate and extrastriate visual cortical areas of the cat. Proc. Natl. Acad. Sci. U. S. A. 88, 6048-6052.

Foxe, J.J., Simpson, G.V., Ahlfors, S.P., 1998. Parieto-occipital approximately $10 \mathrm{~Hz}$ activity reflects anticipatory state of visual attention mechanisms. NeuroReport 9, 3929-3933.

Freedman, D.J., Riesenhuber, M., Poggio, T., Miller, E.K., 2003. A comparison of primate prefrontal and inferior temporal cortices during visual categorization. J. Neurosci. 23, 5235-5246.

Fries, P., Neuenschwander, S., Engel, A.K., Goebel, R., Singer, W., 2001. Rapid feature selective neuronal synchromization through correlated latency shifting. Nat. Neurosci. 4, 194-200.

Fu, K.M., Foxe, J.J., Murray, M.M., Higgins, B.A., Javitt, D.C., Schroeder, C.E., 2001. Attention-dependent suppression of distracter visual input can be cross-modally cued as indexed by anticipatory parieto-occipital alpha-band oscillations. Brain Res. Cogn. Brain Res. 12, 145-152.

Gambini, J.P., Velluti, R.A., Pedemonte, M., 2002. Hippocampal theta rhythms synchronizes visual neurons in sleep and waking. Brain Res. 926, 137-141.

Gho, M., Varela, F.J., 1988. A quantitative assessment of the dependency of the visual temporal frame upon the cortical rhythm. J. Physiol. (Paris) $83,95-101$.

Gosselin, F., Schyns, P.G., 2001. Bubbles: a new technique to reveal the use of visual information in recognition tasks. Vis. Res. 41, 2261-2271.

Gray, C.M., Konig, P., Engel, A.K., Singer, W., 1989. Oscillatory responses in cat visual cortex exhibit inter-columnar synchronization which reflects global stimulus properties. Nature 338, 334-337.

Hopcroft, J.E., Ullman, J.D., 1979. Introduction to Automata Theory, Languages and Computation. Addison-Wesley, Reading, MA.

Klimesch, W., 1999. EEG alpha and theta oscillations reflect cognitive and memory performance: a review and analysis. Brain Res. Brain Res. Rev. 29, 169-195. 
Large, E.W., Jones, M.R., 1999. The dynamics of attending: how people track time-varying events. Psychol. Rev. 106, 119-159.

Lisman, J., Idiart, M.A.P., 1995. Storage of $7 \pm 2$ short-term memories in oscillatory subcycles. Science 267, 1512-1515.

Logothetis, N.K., Pauls, J., Poggio, T., 1995. Shape representation in the inferior temporal cortex of monkeys. Curr. Biol. 5, 552-563.

Nielsen, K.J., Logothetis, N.K., Rainer, G., 2006. Dissociation between local field potentials and spiking activity in macaque inferior temporal cortex reveals diagnosticity-based encoding of complex objects. J. Neurosci. 26, 9639-9645.

Nielsen, K., Rainer, G., 2007. Object recognition: similar visual strategies of birds and mammals. Curr. Biol. 17, R174-R176.

Pare, D., Collins, D.R., Pelletier, J.G., 2002. Amygdala oscillations and the consolidation of emotional memories. Trends Cogn. Sci. 6, 306-314.

Rayner, K., 1998. Eye-movements in reading and information processing: 20 years of research. Psychol. Bull. 124, 372-422.

Ritz, R., Sejnowski, T.J., 1997. Synchronous oscillatory activity in sensory systems: new vistas on mechanisms. Curr. Opin. Neurobiol. 7, 536-546.

Rossion, B., Delvenne, J.-F., Debatisse, D., Goffaux, V., Bruyer, R., Crommelinck, M., Guérit, J.-M., 1999. Spatio-temporal localization of the face inversion effect: an event-related potentials study. Biol. Psychol. 50, 173-189.

Rossion, B., Gauthier, I., Tarr, M.-J., Despland, P., Linotte, S., Bruyer, R., Crommelinck, M., 2000. The N170 occipito-temporal component is enhanced and delayed to inverted faces but not to inverted objects: an electrophysiological account of face-specific processes in the human brain. Neuroreport 11, 1-6.

Rossion, B., Gauthier, I., Goffaux, V., Tarr, M.J., Crommelinck, M., 2002. Expertise training with novel objects leads to left-lateralized face-like electrophysiological responses. Psychol. Sci. 13, 250-257.

Rossion, B., Caldara, R., Seghier, M., Schuller, A.-M., Lazeyras, F., Mayer, E., 2003. A network of occipito-temporal face-sensitive areas besides the right middle fusiform gyrus is necessary for normal face processing. Brain 126, 2381-2395.

Sagiv, N., Bentin, S., 2001. Structural encoding of human and schematic faces: holistic and part-based processes. J. Cogn. Neurosci. 13, 937-951.

Schuller, A.M., Rossion, B., 2001. Spatial attention triggered by eye gaze increases and speeds up early visual activity. NeuroReport 12, 2381-2386.

Schweinberger, S.R., Pickering, E.C., Jentzsch, I., Burton, A.M., Kauffman, J.M., 2002. Cogn. Brain Res. 14, 398-409.

Schyns, P.G., 1998. Diagnostic recognition: task constraints, object information, and their interactions. Cognition 67, 147-179.

Schyns, P.G., Bonnar, L., Gosselin, F., 2002. Show me the features! Understanding recognition from the use of visual information. Psychol. Sci. 13, 402-409.

Schyns, P.G., Jentzsch, I., Johnson, M., Schweinberger, S.R., Gosselin, F., 2003. A principled method for determining the functionality of ERP components. NeuroReport 14, 1665-1669.

Sigala, N., Logothetis, N.K., 2002. Visual categorization shapes feature selectivity in the primate temporal cortex. Nature 415, 318-320.

Singer, W., 1999. Time as coding space? Curr. Opin. Neurobiol. 9, 189-194.

Smith, M.L., Gosselin, F., Schyns, P.G., 2004. Receptive fields for flexible face categorizations. Psychol. Sci. 15, 753-761.

Smith, M.L., Cottrell, G.W., Gosselin, F., Schyns, P.G., 2005. Transmitting and decoding facial expressions. Psychol. Sci. 16, 184-189.

Smith, M.L., Gosselin, F., Schyns, P.G., 2006. Perceptual moments of conscious visual experience inferred from oscillatory brain activity. Proc. Natl. Acad. Sci. U.S.A. 103, 5626-5631.

Tallon-Baudry, C., Bertrand, O., 1999. Oscillatory gamma activity in humans and its role in object representation. Trends Cogn. Sci. 3, 151-162.

Thierry, G., Martin, C.D., Downing, P., Pegna, A.J., 2007. Controlling for interstimulus perceptual variance abolishes N170 face selectivity. Nat. Neurosci. 10, 505-511.

Van Rullen, R., Koch, C., 2003. Is perception discrete or continuous? Trends Cogn. Sci. 7, 207-213.

Varela, F.J., Toro, A., John, E.R., Schwartz, E.L., 1981. Perceptual framing and cortical alpha rhythm. Neuropsychologia 19, 675-686.

Ward, L., 2003. Synchronous neural oscillations and cognitive processes. Trends Cogn. Sci. 7, 553-559. 